\title{
Hypomania Induced by Bifrontal Transcranial Direct Current Stimulation in a Patient with Bipolar Depression
}

\author{
Pei-Chun Chao, Chuan-Chia Chang ${ }^{\bowtie}$, and Hsin-An Chang $\bowtie$ \\ Department of Psychiatry, Tri-Service General Hospital, National Defense Medical Center, Taipei, Taiwan
}

Transcranial direct current stimulation (tDCS), a non-invasive neuromodulation technique, has been increasingly used to treat bipolar depression. Researchers recently noticed the risk of tDCS-emergent mania/hypomania in depressed patients and started to evaluate this risk by launching a meta-analysis. Here we present a female with bipolar II depression who rapidly developed hypomanic switching during bifrontal tDCS.

Psychiatry Investig 2018;15(9):914-915

Key Words Bipolar depression, Transcranial direct current stimulation, Hypomanic switch.

To the Editor,

Transcranial direct current stimulation (tDCS), a non-invasive neuromodulation technique, has been increasingly used to treat bipolar depression. ${ }^{1}$ Researchers recently noticed the risk of tDCS-emergent mania/hypomania in depressed patients and started to evaluate this risk by launching a metaanalysis. ${ }^{2}$ Here we present a female with bipolar II depression who rapidly developed hypomanic switching during bifrontal tDCS.

A 53-year-old female with a 17-year history of bipolar II disorder was hospitalized due to her recurrent major depression episode (MDE) lasting for 7 months. Her medical history was unremarkable. Physical examinations during admission did not reveal any abnormal physical findings. Laboratory tests including urinalysis, complete blood tests including thyroid, renal and liver function were all unremarkable. She had her first hypomanic episode at age 36. Thereafter, she had been hospitalized four times for MDEs. She responded well

Received: February 15, 2018 Revised: June 16, 2018

Accepted: August 11, 2018

$\triangle$ Correspondence: Hsin-An Chang, MD

Department of Psychiatry, Tri-Service General Hospital, No. 325, Cheng-Kung Road, Sec. 2, Nei-Hu District, Taipei, 114, Taiwan, ROC

Tel: +11-886-2-8792-7220, Fax: +11-886-2-8792-7221

E-mail: chang.ha@mail.ndmctsgh.edu.tw

$\triangle$ Correspondence: Chuan-Chia Chang, MD, PhD

Department of Psychiatry, Tri-Service General Hospital, No. 325, Cheng-Kung Road, Sec. 2, Nei-Hu District, Taipei, 114, Taiwan, ROC

Tel: +11-886-2-8792-7220, Fax: +11-886-2-8792-7221

E-mail: changcc@mail.ndmctsgh.edu.tw

(a) This is an Open Access article distributed under the terms of the Creative Commons Attribution Non-Commercial License (https://creativecommons.org/licenses/bync/4.0) which permits unrestricted non-commercial use, distribution, and reproduction in any medium, provided the original work is properly cited. to serial trials of antidepressants in combination with secondgeneration antipsychotics in her previous episodes and had never been treated with electroconvulsive therapy. In the current MDE, she had been treated with daily doses of agomelatine $50 \mathrm{mg}$, trazodone $150 \mathrm{mg}$, olanzapine $5 \mathrm{mg}$ and sedative-hypnotics (estazolam $4 \mathrm{mg}$, lorazepam $2 \mathrm{mg}$, alprazolam $2 \mathrm{mg}$, flurazepam $30 \mathrm{mg}$, midazolam $7.5 \mathrm{mg}$ and zolpidem $20 \mathrm{mg}$ ) consecutively for five months but the response was limited. Thus, she participated in a clinical trial of adjunctive bifrontal tDCS (No. of IRB approval: TSGHIRB-2-103-03002; ClinicalTrials.gov ID: NCT03287037) on May 8, 2017. Direct current (DC) generated by a DC stimulator was bilaterally delivered through a pair of saline-soaked surface sponge electrodes $\left(35 \mathrm{~cm}^{2}\right)$. Stimulation was applied at an intensity of $2 \mathrm{~mA}$ for $20 \mathrm{~min}$, twice-daily, on 5 consecutive weekdays. The anode was placed over the left dorsolateral prefrontal cortex (DLPFC) (centered at electrode F3 of the International EEG 10-20 System) and cathode over right DLPFC (centered at electrode F4). To adhere to the study protocol, her medications remained unchanged throughout the trial. Hypomanic switching occurred after four sessions of tDCS. She showed euphoria, talkativeness, increased energy and decreased need for sleep and was otherwise fully functioning. The 10 sessions of $\mathrm{tDCS}$ course finished and a significant reduction in Hamilton Depression Rating Scale was observed (from 41 to 3). The endpoint assessment showed a score of 11 in the Young Manic Rating Scale. She became euthymic one week after the final session of tDCS and recovered at a 3-month follow-up. From then on, midazolam and zolpidem were discontinued and the doses of agomelatine and olanzapine were tapered 
to $25 \mathrm{mg}$ and $2.5 \mathrm{mg}$ per day, respectively. So far, she has been stabilized for at least 7 months.

Antidepressants-emergent mood switching, which appears frequently in the literature, is crucial for bipolar depression because of its potential for worsening rapid-cycling course. ${ }^{3}$ Hypomanic switching during bifrontal tDCS is an infrequent clinical encounter, reported sparsely in the literature. ${ }^{1}$ The definite mechanism is undetermined. A putative explanation may be modulation of the firing of left DLPFC neurons via activation of sodium/calcium channels and lowering the threshold of postsynaptic depolarization by anodal tDCS. This mechanism of action is associated with the pathophysiology of hypomania/mania and may counteract the effects of mood stabilizers. ${ }^{4}$

Notably, DSM-5 criteria indicate that a hypomanic episode can be sufficiently diagnosed when hypomania symptoms were initially triggered during antidepressant treatment but a full syndrome (not just one or two symptoms) persists beyond the physiological effect of that treatment. There was evidence indicating that the after-effects of tDCS last only for a short duration. ${ }^{5}$ Thus, we cannot exclude the likelihood of naturally occurring hypomania in the case. This possibility has been supported by a recent meta-analysis which suggests that active tDCS was not associated with greater numbers of treatmentemergent mania/hypomania episodes. ${ }^{2}$

The tDCS-emergent hypomania in our case is fast-onset, self-limited and resolves without intervention. The pharmacological agents used to treat her depressive symptoms were thereby reduced after this clinical event. All these are the dis- tinguishing features of our case. It looks like the tDCS-emergent hypomania did more good than harm to our case. Controlled studies are warranted to compare the clinical outcomes between tDCS-associated mood switching and naturally occurring mania/hypomania and to verify whether tDCS can only be used in patients suffering from bipolar depression with their mood stabilizers at anti-manic dosages?

\section{Acknowledgments}

This study was supported in part by grants from the Ministry of Science and Technology of the Taiwanese Government (MOST 106-2314-B-016 -021 -MY3), the Tri-Service General Hospital (TSGH-C105-121) and the National Defense Medical Research (MAB-107-087).

\section{REFERENCES}

1. Donde C, Amad A, Nieto I, Brunoni AR, Neufeld NH, Bellivier F, et al. Transcranial direct-current stimulation (tDCS) for bipolar depression: a systematic review and meta-analysis. Prog Neuropsychopharmacol Biol Psychiatry 2017;78:123-131.

2. Brunoni AR, AH Moffa, B Sampaio-Junior, Gálvez V, Loo CK. Treatment-emergent mania/hypomania during antidepressant treatment with transcranial direct current stimulation (tDCS): a systematic review and meta-analysis. Brain Stimul 2017;10:260-262.

3. El-Mallakh RS, Vohringer PA, Ostacher MM, Baldassano CF, Holtzman NS, Whitham EA, et al. Antidepressants worsen rapid-cycling course in bipolar depression: A STEP-BD randomized clinical trial. J Affect Disord 2015;184:318-321.

4. Goldsmith DR, Wagstaff AJ, Ibbotson T, Perry CM. Spotlight on lamotrigine in bipolar disorder. CNS Drugs 2004;18:63-67.

5. Nitsche MA, Fricke K, Henschke U, Schlitterlau A, Liebetanz D, Lang $\mathrm{N}$, et al. Pharmacological modulation of cortical excitability shifts induced by transcranial direct current stimulation in humans. J Physiol 2003;553:293-301. 